Diabetologe 2018 $\cdot 14: 374-375$

https://doi.org/10.1007/s11428-018-0381-4

(C) Springer Medizin Verlag GmbH, ein Teil von Springer Nature 2018

CrossMark

\title{
Michael Lehrke
}

Klinik für Kardiologie, Pneumologie, Angiologie und Internistische Intensivmedizin (Medizinische Klinik I), Uniklinik RWTH Aachen, Aachen, Deutschland

\section{Diabetes und Herz}

Kardiovaskuläre Erkrankungen bleiben die führende Todesursache von Patienten mit Diabetes. Ihre Vermeidung und Behandlung sollten im Fokus der therapeutischen Bemühungen stehen. Dieses Ziel wird am wirkungsvollsten durch eine kombinierte, multifaktorielle Behandlung aller Risikofaktoren unter Berücksichtigung von Lebensstil, Blutzuckerspiegel, Lipidwerten und Blutdruck erreicht. Als eine ergänzende Säule könnten in Zukunft antiinflammatorische/entzündungsmodulierende Therapieansätze an Bedeutung gewinnen.

In der aktuell veröffentlichten Studie CANTOS („canakinumab antiinflammatory thrombosis outcome study“) wurde erstmalig gezeigt, dass kardiovaskuläre Ereignisse bei Patienten mit hoher inflammatorischer Aktivität (erfasst über ein hsCRP [hochsensitives C-reaktives Protein] > 20 mg/dl ohne Infektfokus) durch eine Inhibition des IL-1Signalwegs (IL: Interleukin) vermieden werden können [1]. Der Nutzen dieser immunmodulierenden Therapie erwies sich als maßgeblich größer als das Risiko der begleitenden Immunsuppression, was im vorliegenden Heft ausführlich unter Bezugnahme auf den Patienten mit Diabetes und dessen metabolische Inflammation diskutiert wird.

Erfreulicherweise verbesserte sich die Evidenz, wie sich kardiovaskuläre Ereignisse über eine blutzuckerspiegelsenkende Therapie vermeiden lassen, in den letzten Jahren deutlich. So wurde eine Vielzahl kardiovaskulärer Endpunktstudien veröffentlicht, die insbesondere eine Überprüfung der kardiovaskulären Sicherheit neuer Diabetesmedikamente zum Ziel hatten. Hierbei wurde nachgewiesen, dass deutliche Unterschiede sowohl zwischen den Substanzklassen als auch innerhalb der Substanzgruppen bestehen. So gelang es in EMPAREG OUTCOME („empagliflozin cardiovascular outcome event trial in type 2 diabetes mellitus patients") erstmals, die Prognose von Patienten mit Diabetes und kardiovaskulären Erkrankungen durch eine empagliflozinabhängige SGLT-2Inhibition (SGLT-2: „,sodium dependent glucose transporter 2") nachhaltig $\mathrm{zu}$ verbessern [2]. In ähnlicher Weise wurde in der LEADER-Studie (LEADER: "liraglutide effect and action in diabetes: evaluation of cardiovascular outcome results") gezeigt, dass eine Aktivierung des GLP-1-Rezeptors (GLP-1: „glucagonlike peptide 1") durch Liraglutid zu einer Verbesserung der Prognose bei Patienten mit kardiovaskulären Erkrankungen führt [3]. Die aktuelle Studienlage hierzu wird in der vorliegenden Ausgabe zusammengefasst und ihre Bedeutung für den Patienten mit Diabetes besprochen.

\section{》) Eine zentrale Säule der kardiovaskulären Prävention ist die Lipidtherapie}

Eine zentrale Säule der kardiovaskulären Prävention bleibt die Lipidtherapie, von der insbesondere Patienten mit Diabetes profitieren. Als primäres Therapieziel sollte für alle von dieser Stoffwechselstörung Betroffenen eine Senkung des LDLCholesterin-Spiegels (LDL: „low density lipoprotein“) auf Werte $<100 \mathrm{mg} / \mathrm{dl}$ bzw., bei Vorliegen zusätzlicher Risikofaktoren, $<70 \mathrm{mg} / \mathrm{dl}$ angestrebt werden. Dies gelingt zumeist über eine Statintherapie, die bei nicht ausreichender Wirksamkeit oder Unverträglichkeiten durch Ezetimib und/oder eine PCSK9-Inhibition (PCSK9: Proproteinkonvertase Subtilisin/Kexin Typ 9) ergänzt werden kann. 
Für welche Patienten PCSK9-Inhibitoren geeignet sind und wie Personen mit Diabetes auf eine solche Therapie reagieren, wird in diesem Heft dargestellt.

Lange Zeit fand die Herzinsuffizienz, als Endstrecke kardiovaskulärer Erkrankungen, bei Patienten mit Diabetes nicht genügend Beachtung. Dies wandelte sich insbesondere in jüngerer Zeit aufgrund der günstigen Wirkung der SGLT-2-Inhibition auf die Herzinsuffizienz. Wie sich der kardiale Metabolismus bei Vorliegen eines Diabetes verändert und über welche Mechanismen die SGLT-2-Hemmung hierauf Einfluss nimmt, wird in der vorliegenden Ausgabe praxisnah diskutiert.

Patienten mit Diabetes weisen häufig Vorhofflimmern auf und sind insbesondere durch thrombembolische Komplikationen gefährdet. Wie sich die diabetologische Stoffwechsellage auf das Herzleitungssystem auswirkt und ob dies therapeutisch beeinflusst werden kann, wird ebenfalls im vorliegenden Band dargestellt.

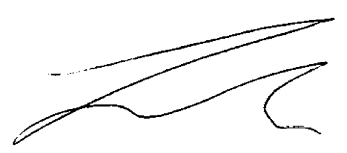

PD Dr. Michael Lehrke

\begin{tabular}{l} 
Korrespondenzadresse \\
$\begin{array}{l}\text { PD Dr. M. Lehrke } \\
\text { Klinik für Kardiologie, } \\
\text { Pneumologie, Angiologie und } \\
\text { Internistische Intensivmedizin } \\
\text { (Medizinische Klinik I), } \\
\text { Uniklinik RWTH Aachen } \\
\text { Pauwelsstraße 30, } \\
\text { 52074 Aachen, Deutschland } \\
\text { mlehrke@ukaachen.de }\end{array}$ \\
\hline
\end{tabular}

Interessenkonflikt. M. Lehrke hat finanzielle Unterstützung für experimentelle und klinische Studien von der Firma Boehringer Ingelheim erhalten und war als Berater für die Firmen Boehringer Ingelheim, SanofiAventis, MSD, AstraZeneca, Lilly, NovoNordisk, Amgen und Bayer und als Referent für die Firmen Boehringer Ingelheim, Sanofi-Aventis, MSD, AstraZeneca, Lilly, NovoNordisk und Bayer tätig.

\section{Literatur}

1. Ridker PM, Everett BM, Thuren T, MacFadyen JG, Chang WH, Ballantyne C, Fonseca F, Nicolau J, Koenig W, Anker SD, Kastelein JJP, Cornel JH, Pais P, Pella D, Genest J, Cifkova R, Lorenzatti A, Forster T,
Kobalava Z, Vida-Simiti L, Flather M, Shimokawa H, Ogawa H, Dellborg M, Rossi PRF, Troquay RPT, Libby P, Glynn RJ, Group CT (2017) Antiinflammatory Therapy with Canakinumab for Atherosclerotic Disease. NEngl J Med 377:1119-1131

2. Zinman B, Wanner $C$, Lachin JM, Fitchett $D_{\text {, }}$ Bluhmki E, Hantel S, Mattheus M, Devins T, Johansen OE, Woerle HJ, Broedl UC, Inzucchi SE, Investigators E-RO (2015) Empagliflozin, Cardiovascular Outcomes, and Mortality in Type 2 Diabetes. NEngl J Med 373:2117-2128

3. Marso SP, Daniels GH, Brown-Frandsen K, Kristensen P, Mann JF, Nauck MA, Nissen SE, Pocock S, Poulter NR, Ravn LS, Steinberg WM, Stockner M, Zinman B, Bergenstal RM, Buse JB, Committee LS Investigators LT (2016) Liraglutide and Cardiovas cular Outcomes in Type 2 Diabetes. N Engl J Med 375:311-322

\section{Künstliche Pankreas bewährt sich im Spital}

Die Einstellung des Blutzuckers im Spital ist eine grosse Herausforderung: akute Krankheit, Änderungen in der Ernährung, Einnahme von Medikamenten und medizinische Behandlungen führen zu raschen Änderungen des Insulinbedarfs und des Blutzuckers. Schweizer und englische Wissenschaftler erforschten inwiefern das künstliche Pankreas das Diabetesmanagement im Spital verbessern kann. «Das künstliche Pankreas hat sich in unserer Studie bewährt», so eine der Schweizer Forscher. «Im Vergleich zur herkömmlichen Therapie wiesen Patientinnen und Patienten fast viermal öfter einen idealen Blutzuckerwert auf. Werte oberhalb des Zielbereichs wurden um ein Viertel reduziert und der Blutzuckerverlauf zeigte signifikant weniger Schwankungen. Der grosse Vorteil des künstlichen Pankreas liegt in der prompten Reaktion auf einen veränderten Insulinbedarf.»

Insgesamt wurden 136 stationäre Patientinnen und Patienten mit Typ-2-Diabetes rekrutiert. Nach dem Zufallsprinzip erhielten die Studienteilnehmenden entweder die herkömmliche Therapie mit manuellem Nachmessen und Insulininjektionen oder die Blutzuckereinstellung erfolgte mit dem künstlichen Pankreas. Das künstliche Pankreas besteht aus einem kontinuierlichen Glukosesensor, einer Insulinpumpe und einem Kontrollalgorithmus, der die Insulinabgabe je nach Blutzuckerwert bedarfsgerecht reguliert.

Folgestudien werden die Auswirkung der verbesserten Blutzuckerkontrolle durch das künstliche Pankreas auf den Genesungsverlauf der Patientinnen und Patienten untersuchen.

Originalpunlikation: Bally L. et al. (2018) Closed-Loop Insulin Delivery for Glycemic Control in Noncritical Care. N Engl J Med. (doi: 10.1056/NEJMoa 1805233)

Quelle: Inselspital, Universitätsspital Bern, www.insel.ch 